\title{
Maize provitamin A carotenoids, current resources, and future metabolic engineering challenges
}

\author{
Eleanore T. Wurtzel ${ }^{1,2}{ }^{*}$, Abby Cuttriss $^{1,3}$ and Ratnakar Vallabhaneni ${ }^{1,2}$ \\ 1 Department of Biological Sciences, Lehman College, The City University of New York, NY, USA \\ ${ }^{2}$ The Graduate School and University Center of the City University of New York, New York, NY, USA \\ ${ }^{3}$ Department of Biology, University of Hawaii, Hilo, HI, USA
}

\section{Edited by:}

William David Nes, Texas Tech

University, USA

\section{Reviewed by:}

Fumiya Kurosaki, University of

Toyama, Japan

Ralf Welsch, Albert-Ludwigs-

University, Germany

*Correspondence:

Eleanore T. Wurtzel, Department of Biological Sciences, Lehman College,

The City University of New York, 250

Bedford Park BIvd. West, Bronx, New

York 10468, USA.

e-mail:wurtzel@lehman.cuny.edu
Vitamin A deficiency is a serious global health problem that can be alleviated by improved nutrition. Development of cereal crops with increased provitamin A carotenoids can provide a sustainable solution to eliminating vitamin A deficiency worldwide. Maize is a model for cereals and a major staple carbohydrate source. Here, we discuss maize carotenogenesis with regard to pathway regulation, available resources, and current knowledge for improving carotenoid content and levels of provitamin A carotenoids in edible maize endosperm. This knowledge will be applied to improve the nutritional composition of related Poaceae crops. We discuss opportunities and challenges for optimizing provitamin A carotenoid biofortification of cereal food crops.

\section{Keywords: carotenoids, maize, vitamin A, metabolic engineering, endosperm, Poaceae, beta-carotene}

\section{VITAMIN A DEFICIENCY}

The FAO estimates that 800 million people are chronically hungry, a problem that is most severe in sub-Saharan Africa, but stretches across the globe to include regions of Asia, the Pacific, Latin America, the Caribbean, and North Africa (FAO, 2000). Vitamin A deficiency in these nations is responsible for a number of disorders that range from impaired iron mobilization, growth retardation, and blindness to a depressed immune response, increased susceptibility to infectious disease and increased childhood mortality (Sommer and Davidson, 2002; WHO, 2009).

\section{MAIZE, A MAJOR FOOD CROP AND MODEL FOR NUTRITIONAL IMPROVEMENT}

The starchy endosperm tissue of cereal crops contributes a major portion of energy intake from the human diet but is typically of low provitamin A nutritional value. Provitamin A carotenoid biofortification of cereal crops would have a global impact on human health. Rice (Oryza sativa) is the most significant worldwide carbohydrate source, but does not accumulate any seed carotenoids. Rice biofortification could only be accomplished by transgenic approaches (Ye et al., 2000; Paine et al., 2005). Sorghum (Sorghum bicolor) is a major staple crop grown in semiarid regions due to its drought tolerance, which makes it a good candidate for biofortification. Yellow endosperm varieties contain provitamin A carotenoids and diverse collections of sorghum landraces have been analyzed to quantify pigment diversity (Fernandez et al., 2009). Wheat (Triticum aestivum) endosperm color is an important agronomic trait, however there is limited natural variation in this tissue (Howitt et al., 2009). Maize (corn, Zea mays) is the predominant food staple in much of sub-Saharan Africa and Latin America, regions that are also plagued by vitamin A deficiency (Sommer and Davidson, 2002).
The significant variation in carotenoid content and composition of maize suggests that maize diversity may hold clues as to the target genes that could be manipulated by breeding or transgenics for improvement of cereal crop provitamin A content (Harjes et al., 2008).

Maize is an essential staple cereal crop that naturally accumulates carotenoids in the edible seed endosperm, and is thus an obvious target for biofortification projects. Maize is also a valuable model for other grass species due to historical collections of carotenoid mutants, genome sequence, and other molecular resources. Maize germplasm resources exhibit wide genetic diversity (Liu et al., 2003) with corresponding variation in carotenoid profiles (Harjes et al., 2008), features that are useful for investigating pathway regulation and generating breeding alleles. The close evolutionary relationship between maize and other food crops in the Poaceae provides an opportunity for using genome synteny to identify new maize targets for provitamin A improvement to gene homologs in other grass species.

\section{LOCALIZATION OF CAROTENOID BIOSYNTHESIS}

Carotenoid pigments are hydrophobic C40 isoprenoids that are synthesized in plant plastids, where they undergo a series of enzymatic modifications that impart different spectral properties and thus colors. Carotenoid biosynthetic enzymes are encoded by nuclear genes, and the proteins must be imported into plastids. Carotenoids that accumulate in cereal endosperm tissue are synthesized in amyloplasts, plastids that are specialized for storage of starch granules (Kirk and Tiliney-Bassett, 1978).

At least part of the biosynthetic pathway is associated with plastid membranes in maize endosperm (Li et al., 2008b). Proteomic analysis in plastids of other plants revealed sites of some but not all carotenoid enzymes in chloroplasts and chromoplasts 
(Ytterberg et al., 2006; Joyard et al., 2009). However, metabolon organization is not well described and is further complicated by differences in plastid membrane organization. Chloroplasts possess a double envelope membrane and internal thylakoid membrane system whereas endosperm amyloplasts have only the double envelope membrane. Given that the endosperm and leaf plastids are architecturally distinct, further research on pathway localization in plastids is a prerequisite for optimizing carotenoid metabolic engineering efforts. An understanding of the biosynthetic pathway enzymes and associated genes will facilitate engineering of enhanced levels of the pathway intermediates that are provitamin A.

\section{THE CAROTENOID BIOSYNTHETIC PATHWAY}

The plant carotenoid biosynthetic pathway has been well characterized after decades of molecular genetic analyses (Cuttriss et al., 2011). In maize seed endosperm, the primary carotenoids that accumulate in diverse cultivars are either lutein or zeaxanthin or a combination of both (see Figure 1). Provitamin A compounds are biosynthetic pathway intermediates and therefore usually not the predominant carotenoids in endosperm, the target of provitamin A biofortification. Vitamin A (all-trans-retinol) is a $\mathrm{C}_{20}$ enzymatic cleavage product made in humans from plant carotenoids containing an unmodified $\beta$-ring (Von Lintig, 2010). $\alpha$-carotene and $\beta$-cryptoxanthin have provitamin A potential, due to their single
A

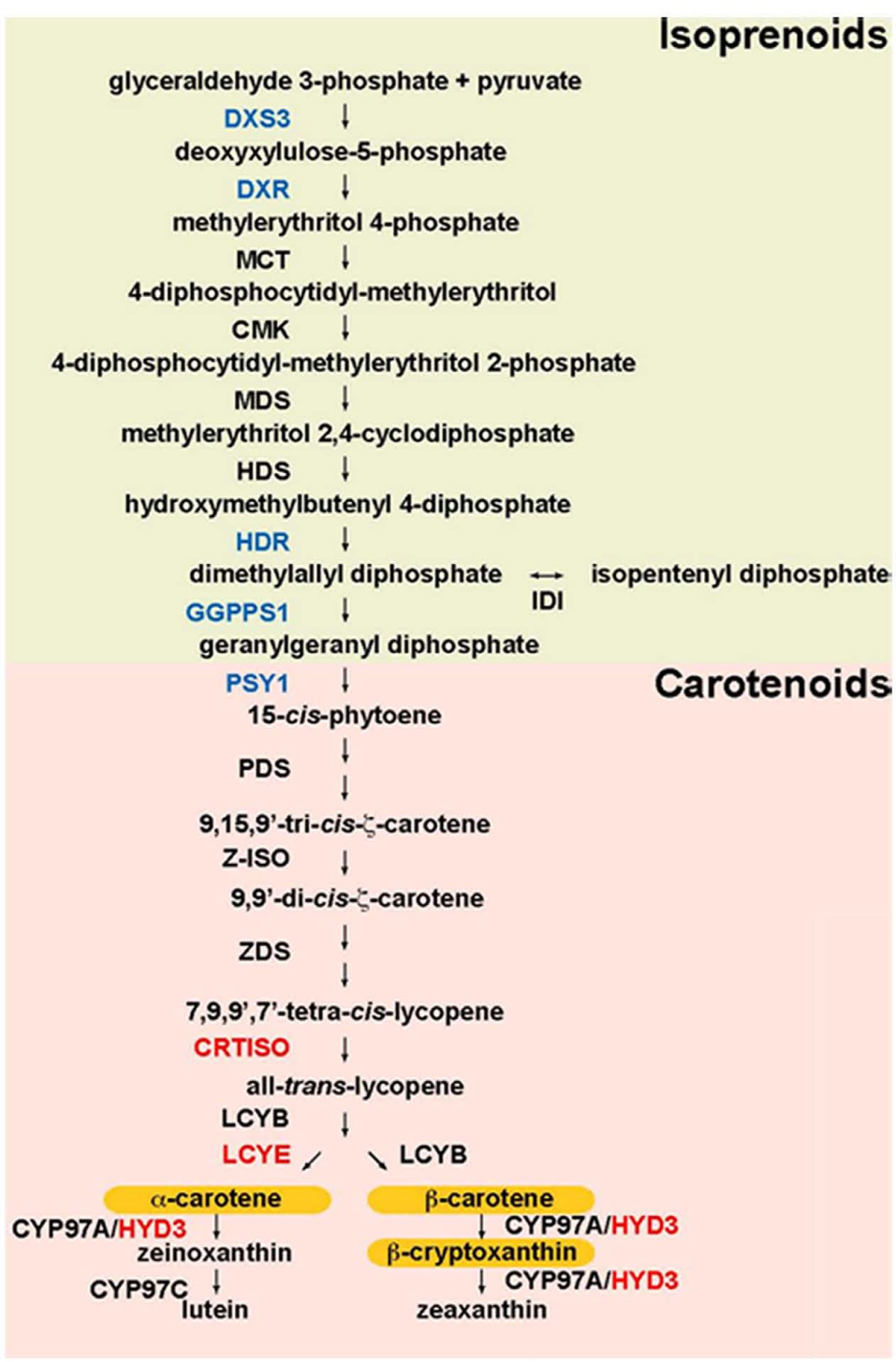

B

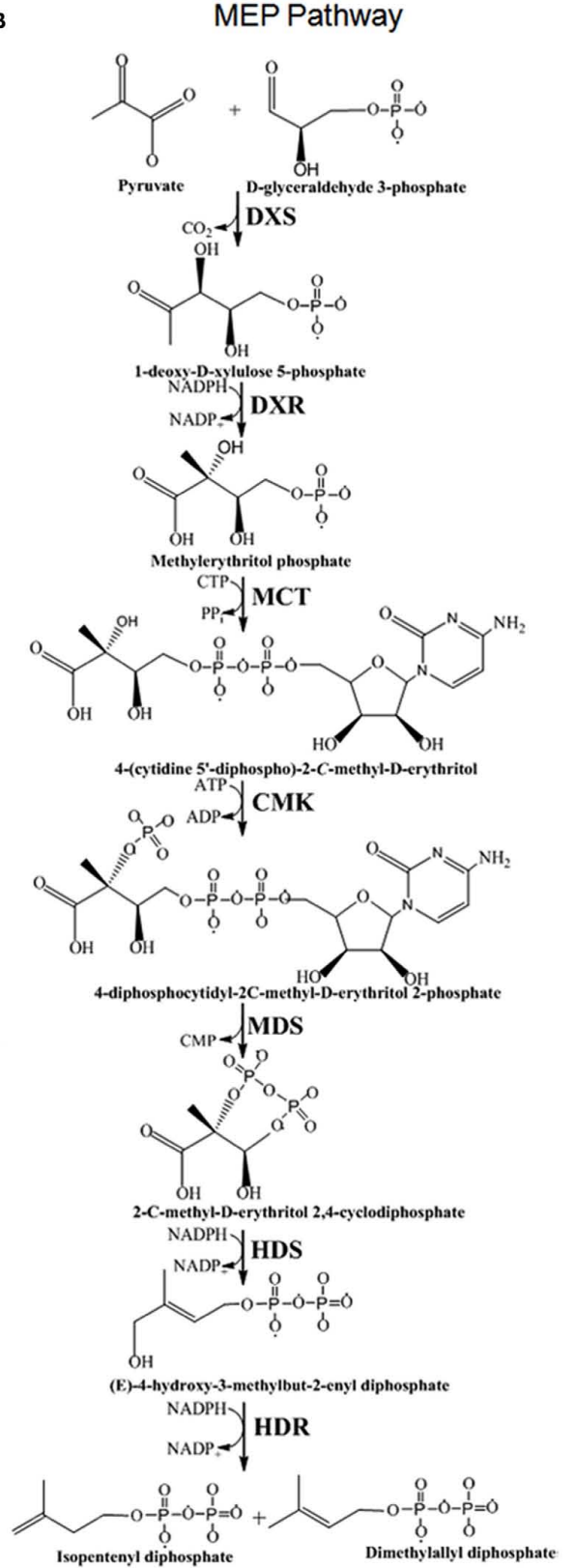

FIGURE 1 | Continued 
c

GGPP Biosynthesis

(3:1)<smiles></smiles>

IPP (C5)

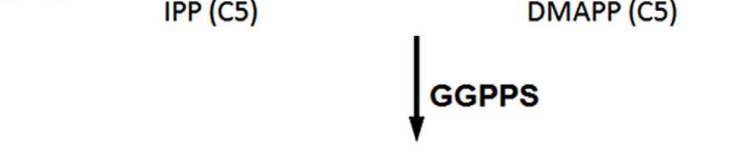

$\overbrace{\text { GGPP (C2O) }}^{\mathrm{CH}_{2} \mathrm{O} \cdot \mathrm{P} P \mathrm{P}}$

Pathways for carotenoids, gibberellins, tocopherols, quinones, chlorophyll phytol chain and others
D

\section{Carotenoid biosynthesis until lycopene}

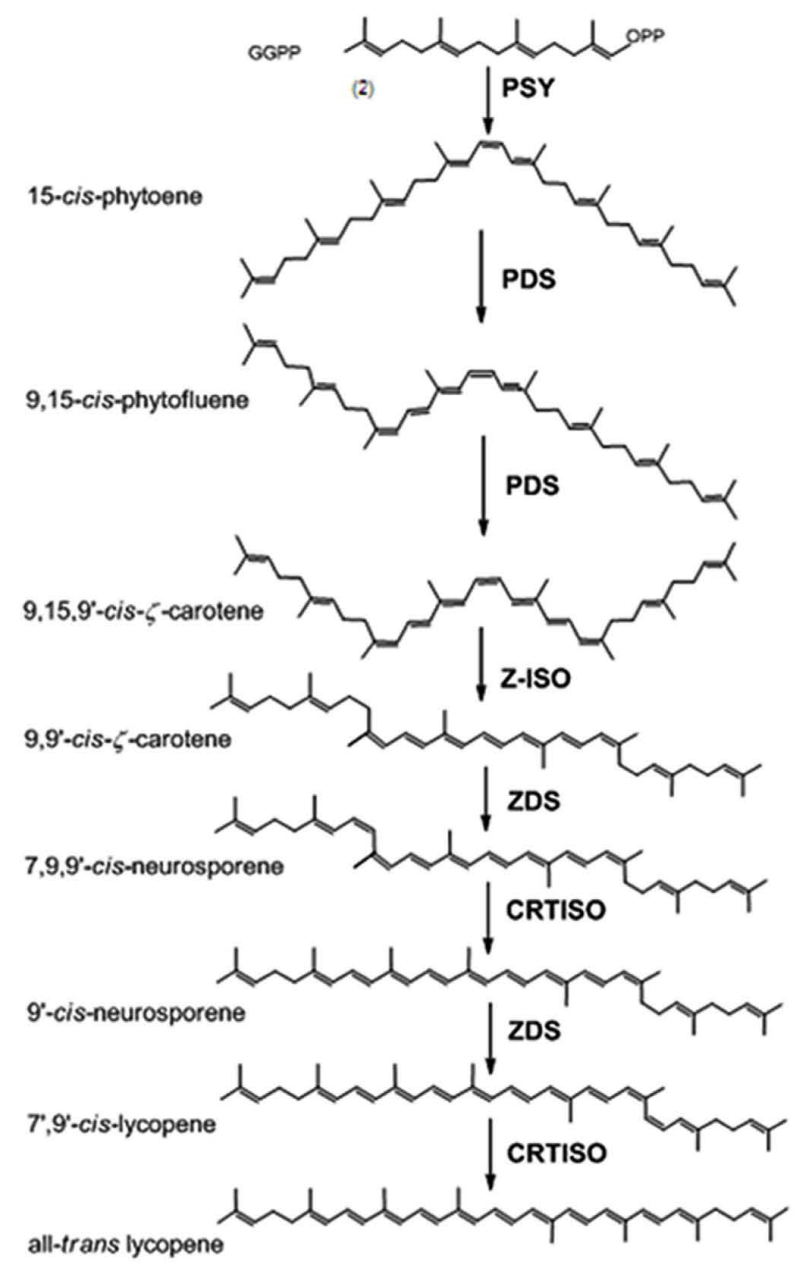

FIGURE 1 | Continued

unmodified $\beta$-ring, but $\beta$-carotene is the most efficient source, as two retinol molecules may be derived from each $\beta$-carotene molecule.

The plastid-localized methylerythritol 4-phosphate (MEP) pathway (Rodriguez-Concepcion, 2010) supplies isoprenoid precursors for carotenoids; glyceraldehyde-3-phosphate and pyruvate are combined to form deoxy-D-xylulose 5-phosphate (DXP), a reaction catalyzed by DXP synthase (DXS), and a number of steps are then required to form geranylgeranyl diphosphate (GGPP), the precursor to carotenoid biosynthesis (Lichtenthaler, 1999) as well as to other biosynthetic pathways. The first carotenoid, phytoene, is produced by the condensation of two GGPP molecules, a reaction that is catalyzed by phytoene synthase (PSY). Two desaturases (PDS, phytoene desaturase; ZDS, $\zeta$-carotene desaturase) and two isomerases (Z-ISO, $\zeta$-carotene isomerase; CRTISO, carotenoid isomerase) introduce a series of double bonds and alter the isomer state of each biosynthetic intermediate to produce all-trans-lycopene. At this point the main biosynthetic pathway branches, depending on cyclization activity. Asymmetric cyclization of lycopene by both $\varepsilon$ - and $\beta$-lycopene cyclases (LCYE and LCYB, respectively) produces $\alpha$-carotene with one $\varepsilon$ - and one $\beta$-ionone ring (Cunningham and Gantt, 2001). Symmetric cyclization by LCYB yields $\beta$-carotene, with two unmodified $\beta$ ionone rings. Hydroxylation of the carotene $\beta$-ionone ring by one of two classes of structural distinct carotene hydroxylase enzymes eliminates provitamin A potential. The hydroxylated carotenes include the non-provitamin A xanthophylls, lutein, and zeaxanthin, which may be further modified to other xanthophylls, some of which are cleaved to form ABA (North et al., 2007).

\section{MAIZE GENETICS OF CAROTENOGENESIS}

Early maize mutant analyses were responsible for much of our understanding of the biosynthesis of carotenoids and the relationship of these pigments with the biogenesis of plastid ultrastructure (Robertson et al., 1966; Treharne et al., 1966; Robertson, 1975; Neill et al., 1986) (Table 1). Carotenoid biosynthesis occurs during seed development (Li et al., 2008b) and the accumulation of 
E

\section{Carotenoid biosynthesis (from lycopene to carotenes and xanthophylls)}

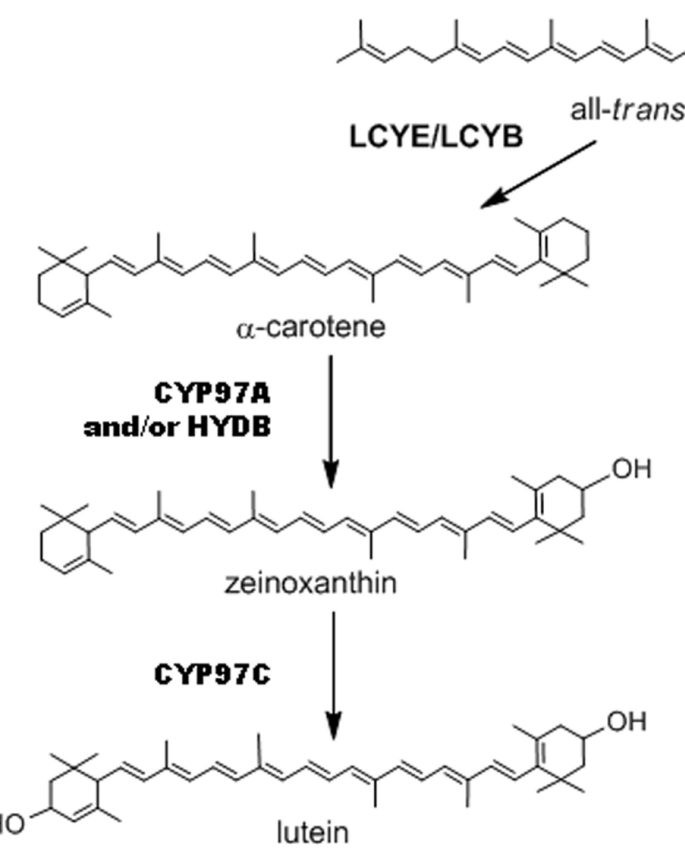

FIGURE 1 |The biosynthetic pathway and challenges for provitamin $A$ metabolic engineering. (A) Regulatory points; (B-E) Structures of carotenoids and their precursors. Pro-vitamin A carotenoids are highlighted in orange. Enzymes with transcript abundance and/or allelic polymorphisms that positively associate with provitamin A carotenoid content are indicated in blue, and those with negative correlation in red (based on Harjes et al., 2008; Li et al., 2008b; Vallabhaneni and Wurtzel, 2009; Vallabhaneni et al., 2009, 2010; Yan et al., 2010). Not shown are CCD1 and ZEP for which transcripts levels are inversely associated with carotenoid content. CMK,

4-diphosphocytidyl-methylerythritol kinase; CRTISO, carotenoid isomerase;

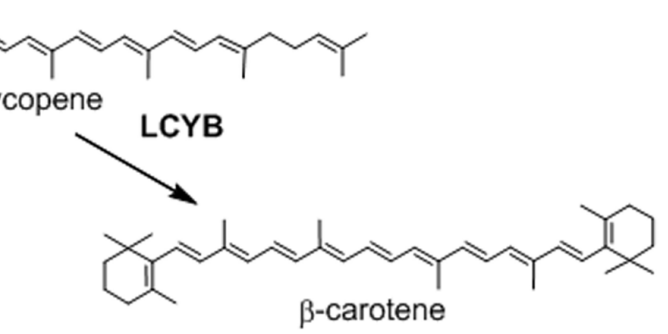

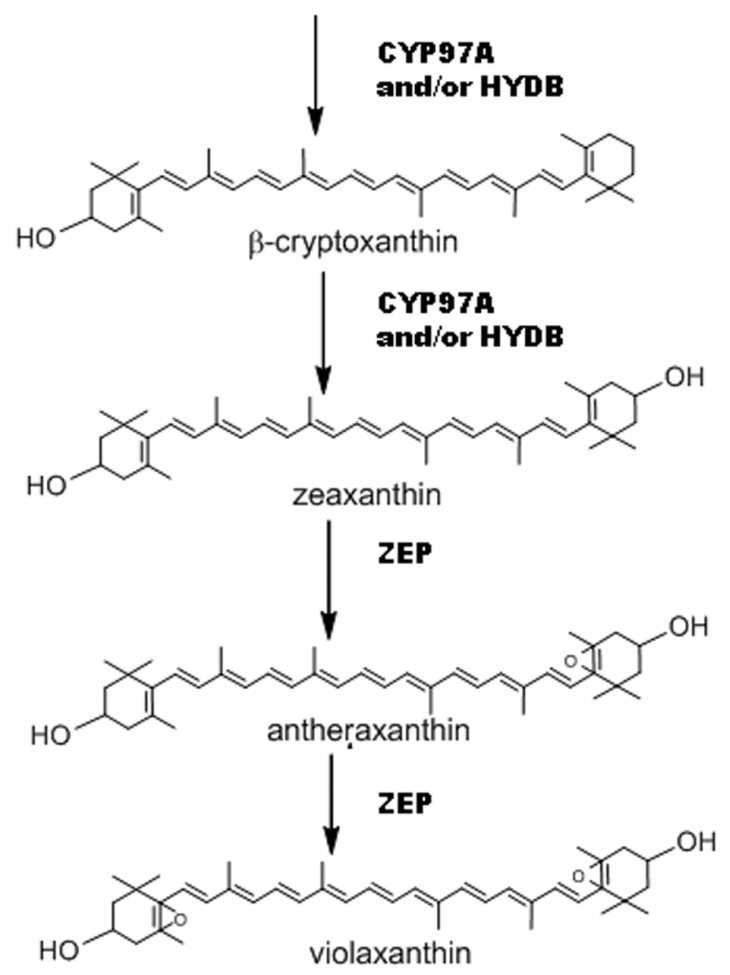

CYP97A/HYD3, $\beta$-hydroxylase; CYP97C, $\varepsilon$-hydroxylase; DXR, deoxyxylulose 5-phosphate reductoisomerase; DXS3, deoxyxylulose 5-phosphate synthase 3; GGPPS1, geranylgeranyl diphosphate synthase 1; HDR, hydroxymethylbutenyl 4-diphosphate reductase; HDS, hydroxymethylbutenyl 4-diphosphate synthase; IDI, isopentenyl diphosphate isomerase; LCYB, $\beta$-cyclase; LCYE, $\varepsilon$-cyclase; MCT, methylerythritol 4-phosphate cytidylyltransferase; MDS, methylerythritol 2,4-cyclodiphosphate synthase; PDS, phytoene desaturase; PSY1, phytoene synthase $1 ; \mathrm{ZDS}, \zeta$-carotene desaturase; Z-ISO, 15-cis- $\zeta$-carotene isomerase; CCD1, carotene cleavage dioxygenase 1 ; ZEP, zeaxanthin epoxidase. carotenoids imparts a yellow-orange color to the endosperm, an easily scored phenotype. Genetic loci controlling carotenogenesis include alleles with recessive and dominant phenotypes. There are also duplicate factors and "modifier genes" (for which the underlying genes are largely unknown) that function together to control carotenoid phenotypes in the seed and seedling. In general, mutations blocking function of the pathway structural genes are recessive and lethal as the absence of carotenoids in seedlings leads to aberrant chloroplast development. "Mutant" seeds have white endosperm in comparison to the "normal" yellow endosperm. The mutant seeds may also exhibit a viviparous phenotype due to precocious germination in the absence of the apocarotenoid abscisic acid (ABA). If the pathway is blocked midway, biosynthetic intermediates accumulate throughout the plant.

As early as 1940, the yellow1 ( $y 1)$ locus was found to have a gene dosage effect on seed carotenoid content (Randolph and 
Table 1 | Maize carotenoid candidate genes, quantitative trait loci, and phenotypic loci.

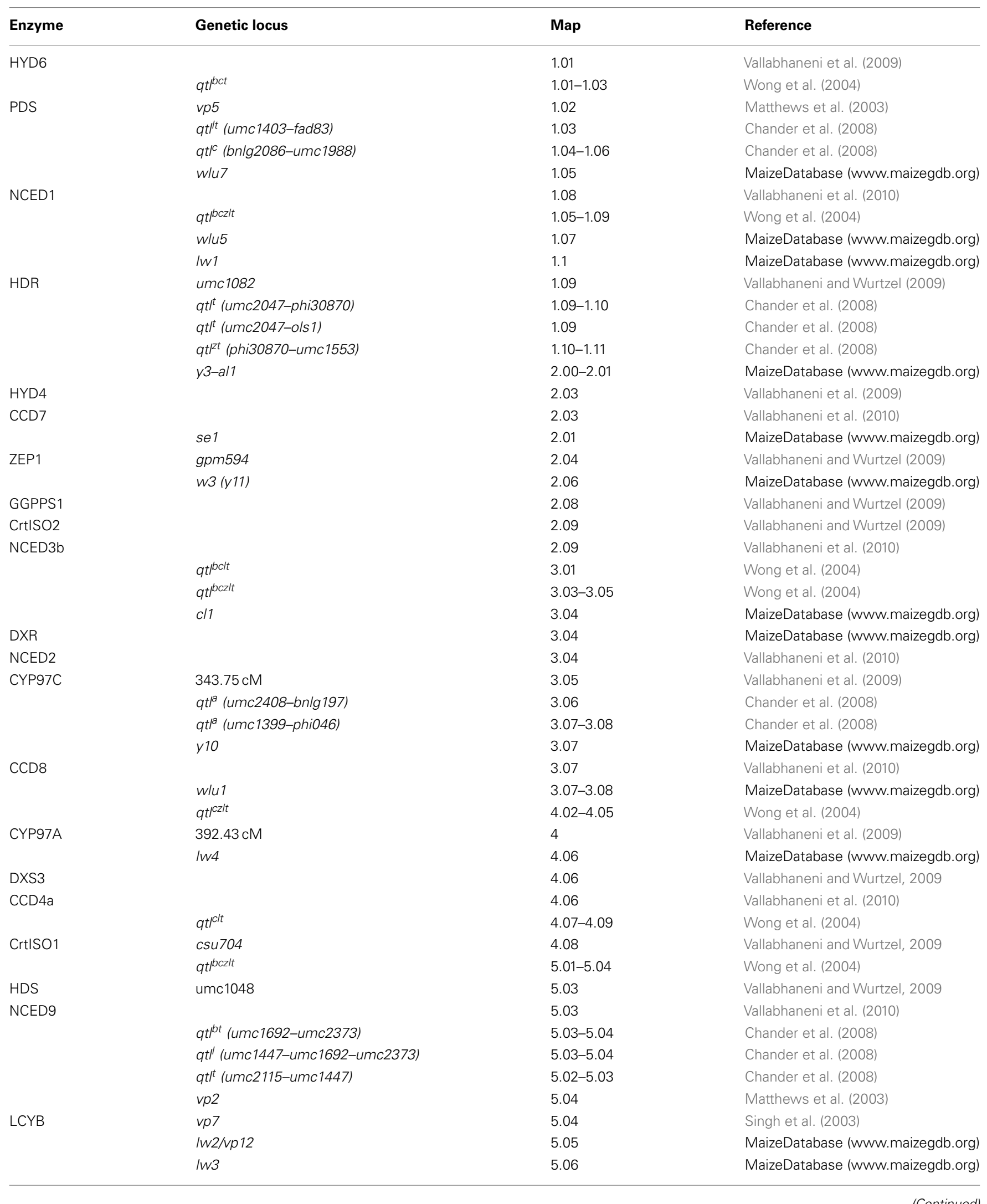

(Continued) 
Table 1 | Continued

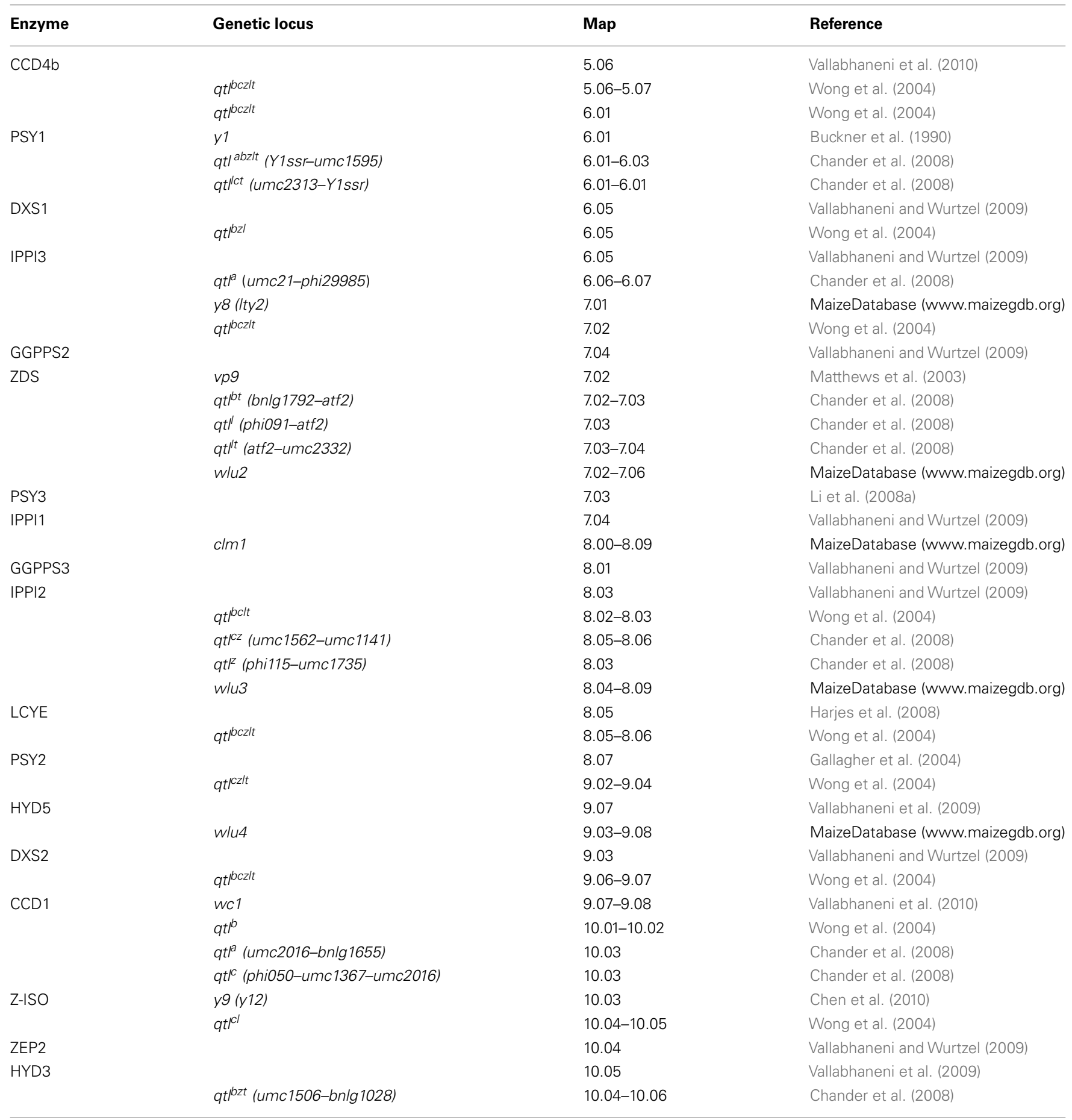

Chromosome locations (Map) are indicated for each carotenoid locus representing a biosynthetic gene, quantitative trait locus (QTL), or known genetic (phenotypic)

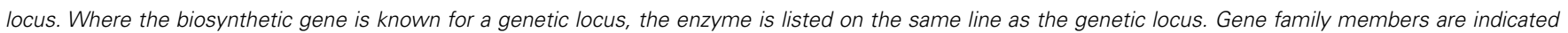

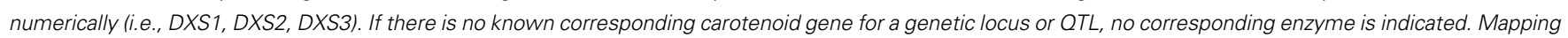
references are cited for the structural gene loci or in order of the gene and genetic locus, where applicable. QTL, quantitative trait locus for enhanced: a, $\alpha$-carotene; b, $\beta$-carotene; $c, \beta$-cryptoxanthin; $z$, zeaxanthin; I, lutein; or $t$, total carotenoids in the seed endosperm.

Hand, 1940) and thus control of pathway flux; three copies of the dominant $Y 1$ allele in the triploid endosperm conditioned the most yellow seeds (endosperm) in contrast to homozygous $y 1$ seeds. White $y 1$ endosperm is an ancestral trait shared with teosinte, the wild progenitor of maize. Cloning and subsequent sequence analyses identified the $Y 1$ gene as encoding PSY1, the 
first enzyme of the biosynthetic pathway (Buckner et al., 1996). Curiously, the yellow $Y 1$ seed accumulates carotenoids because of a gain of function mutation, which came at the loss of photoregulation in green tissue ( $\mathrm{Li}$ et al., 2009). In contrast, the white endosperm $y 1$ allele has maintained photoregulation in green tissue. This alteration in PSY1 regulation across tissues, depending upon $y 1$ allele, highlights the potential ramifications of allele selection for breeding when we do not fully understand the role of gene family members with regard to carotenogenesis in different tissues. More detailed understanding of carotenoid gene families and their respective alleles will strengthen future predictive engineering strategies.

Recently, it was discovered that the maize $y 9$ locus encodes $\zeta$-carotene isomerase (Z-ISO), a previously unknown pathway enzyme that is necessary for carotenogenesis in all plants (Chen et al., 2010). Without Z-ISO function, provitamin A carotenoids cannot be produced in the endosperm, the target tissue for biofortification. The importance of Z-ISO for endosperm carotenogenesis might explain a cluster of Quantitative Trait Loci (QTL) for seed carotenoids (Table 1), five of which were found within $15 \mathrm{cM}$ of the $y 9$ locus (Chander et al., 2008). Another locus affecting seed carotenogenesis, white cap1 ( $w c 1$ ), for which dominant alleles deplete endosperm carotenoids, was recently shown to be the map location (Table 1) for a carotenoid cleavage enzyme CCD1 (Vallabhaneni et al., 2010).

A detailed study of recombinant inbred lines identified more than 30 Quantitative Trait Loci (QTL; Table 1) for carotenoid content and composition (Wong et al., 2004; Chander et al., 2008), some of which are closely linked to biosynthetic pathway structural genes, such as $y 1$ or $y 9$ (Li et al., 2007). The number of QTL outnumber pathway structural genes and represent unknown genes that influence seed carotenoid content and composition. These QTL could lead to novel regulatory genes and other targets for future breeding efforts.

Gene duplication is a common feature in maize and for the maize carotenoid biosynthetic pathway. Carotenoid gene paralogs have been mapped in maize (Table 1), though the role of many duplicates is yet to be determined, which adds to the complexity of pathway regulation in different tissues. Genes encoding carotenoid enzymes are also duplicated in rice, wheat and sorghum (Table 2). As structural genes have been isolated over the years, they have been mapped to chromosome location to determine which known genetic loci might correspond to these structural genes. There are still a number of loci for which alleles affect levels of carotenoids and these loci do not correspond to known structural genes. Such loci represent additional opportunities for gene discovery for controlling seed carotenogenesis.

\section{REGULATION OF SEED CAROTENOID ACCUMULATION}

To improve the provitamin A potential of cereal seeds, the challenges are to increase total carotenoid content, optimize provitamin A composition, limit degradation of beneficial carotenoids, and control carotenoid sequestration. Approaches that may overcome these challenges are outlined below. These efforts have focused on elucidating factors that contribute to provitamin A accumulation in the endosperm through analysis of pathway regulatory points and timing of gene expression for key enzymes. This research has led to new tools and knowledge to help breed higher provitamin A cereal crops.

\section{QUANTITATIVE CONTROL OF CAROTENOID CONTENT THROUGH MAXIMIZING PATHWAY FLUX}

The first step in determining provitamin-A potential is to maximize biosynthetic flux and thus total carotenoid synthesis. PSY catalyzes the first committed biosynthetic step, and is encoded by three genes in maize and in other cereal crops such as rice and wheat (Palaisa et al., 2003; Li et al., 2008b; Welsch et al., 2008; Howitt et al., 2009; Chaudhary et al., 2010). PSY1 is responsible for endosperm carotenogenesis but also plays a role in carotenogenesis in other tissues (Gallagher et al., 2004; Li et al., 2008a,b). The various PSY paralogs respond differently to abiotic stimuli and have unique tissue specificities though their function remains redundant. In addition to its role in endosperm, PSY1 is needed for carotenogenesis related to thermotolerance in photosynthetic tissue (Li et al., 2008b). Salt and drought induced PSY3 transcript abundance in maize roots, and correlated with increased carotenoid flux and ABA in maize roots (Li et al., 2008a). Similar responses were observed for the rice PSY homologs (Welsch et al., 2008), confirming that maize findings can be applied to other cereal biofortification projects and that each member of a conserved gene family has a specific role. QTL analysis determined that PSY1 was responsible for 6.6-27.2\% of phenotypic variation in seed carotenoid content (Chander et al., 2008), suggesting the contribution of additional unknown gene targets.

While PSY is clearly a significant flux determinant, other steps in the pathway are likely to have an effect on the size of the carotenoid pool. To this end, a maize diversity collection was analyzed to reveal those regulatory points and to determine the correlation between carotenoid content and biosynthetic gene transcript levels. A core germplasm collection representing extremes of seed carotenoid composition was created based on carotenoid profiles of 150 maize lines spanning $80 \%$ of maize genetic diversity. Statistical analysis of transcript levels was used to identify specific gene family members that influence carotenoid content and composition and the time during endosperm development when this effect was seen (Li et al., 2008b; Vallabhaneni and Wurtzel, 2009; Vallabhaneni et al., 2009). PSY1 was used to validate the approach. The comprehensive analysis of the maize carotenoid pathway gene families led to discovery of a number of new targets for endosperm carotenoid biofortification.

Rate-limiting steps were identified in both the biosynthesis of carotenoids and the supply of precursor isoprenoids, thus identifying potential metabolic engineering targets (Vallabhaneni and Wurtzel, 2009). Multiple control points both within the carotenoid pathway and MEP precursor pathway were identified in maize, and the timing of gene expression was found to be critical in determining carotenoid content. Transcript levels of CrtISO and ZEP1 and ZEP2 inversely correlated with seed carotenoid content, whereas several genes required to produce isoprenoid precursors showed positive correlation, including DXS3, DXR, HDR, and GGPPS1 (Vallabhaneni and Wurtzel, 2009). The genes identified in this study offer new opportunities for breeding and transgenic fortification. The timing of gene expression is critical in determining carotenoid composition, as expression 
Table 2 | Map positions of candidate genes encoding carotenoid biosynthetic and degradative enzymes in Zea mays, Oryza sativa, Triticum aestivum, and Sorghum bicolor.

\begin{tabular}{|c|c|c|c|c|c|c|c|c|}
\hline \multirow[t]{2}{*}{ Enzyme } & \multicolumn{2}{|c|}{ Maize (Zea mays) } & \multicolumn{2}{|c|}{ Rice (Oryza sativa) } & \multicolumn{2}{|c|}{ Wheat (Triticum aestivum) } & \multicolumn{2}{|c|}{ Sorghum (Sorghum bicolor) } \\
\hline & Chr. bin & $\begin{array}{l}\text { Reference/ } \\
\text { GenBank }\end{array}$ & Chr. bin & $\begin{array}{l}\text { Reference/ } \\
\text { GenBank }\end{array}$ & Chr. bin & $\begin{array}{l}\text { Reference/ } \\
\text { GenBank }\end{array}$ & Chr. Bin and (qtl) & $\begin{array}{l}\text { Reference/ } \\
\text { phytozome }\end{array}$ \\
\hline DXS1 & 6.05 & AY110050 & 5 & AK064944 & & & 9 & Sb09g020140 \\
\hline DXS2 & 9.03 & AY946270 & 7 & AK100909 & & & 2 & Sb02g005380 \\
\hline DXS3 & 4.06 & AY104478 & 6 & AK121920 & & & 10 & Sb10g002960 \\
\hline CMK & 3.06 & BG354410 & 1 & AK067589 & & & 3 & Sb03g037310 \\
\hline MDS & & CD974184 & 2 & AK060238 & & & 4 & 04g031830 \\
\hline HDS & 5.03 & AC198323 & 2 & AK120769 & $6 \mathrm{D}$ & BE585702 & 4 & Sb04g025290 \\
\hline HDR & 1.09 & DR789385 & 3 & AK062113 & & & 1 & Sb01g009140 \\
\hline IPPI1 & 7.04 & AF330034 & 7 & AK065871 & $1 \mathrm{~A}$ & CD452500 & 2 & Sb02g035700 \\
\hline GGPPS2 & 7.04 & EF417574 & 1 & AP002865 & & & & \\
\hline GGPPS3 & 8.01 & AC211188 & & & & & 3 & Sb03g009380 \\
\hline PSY1 & 6.01 & AY324431 & 6 & AY445521 & 7AL, 7BL, 7A & $\begin{array}{l}\text { Yellow pigment } \\
\text { qtl }\end{array}$ & $\begin{array}{l}\text { 10b, } 0.0 \mathrm{cM} \text {, qtl } \\
\text { (color; } p \leq 0.0001 \text { ) }\end{array}$ & Sb10g031020 \\
\hline PSY2 & 8.07 & AY325302 & 12 & AY773943 & $5 A, 5 B$ & $\begin{array}{l}\text { Either PSY } 2 \text { or } \\
\text { PSY3 }\end{array}$ & 8 & Sb08g022310 \\
\hline PSY3 & 7.03 & DQ372936 & 9 & FJ214953 & & & $2,165.9 \mathrm{cM}$ & Sb02g032370 \\
\hline PDS & 1.02 & L39266 & 3 & AF049356 & $4 \mathrm{~A}, 4 \mathrm{~B}$ & & $6,16.9 \mathrm{cM}$ & Sb06g030030 \\
\hline Z-ISO & 10.03 & ВТ036679 & 12 & BAF29644 & & & 8 & Sb08g014610 \\
\hline ZDS & 7.02 & AF047490 & 7 & AK065213 & $2 \mathrm{~A}, 2 \mathrm{~B}$ & & $2,37.1 \mathrm{cM}$ & Sb02g006100 \\
\hline CrtISO1 & 4.08 & $\begin{array}{l}\text { CC749876, } \\
\text { AC218991 }\end{array}$ & 11 & AC108871 & & & 5 & Sb05g022240 \\
\hline HYD3 & 10.05 & AY844958 & & & & & & \\
\hline HYD4 & 2.03 & AC196442 & 4 & AL606624 & & & 6 & Sb06g026190 \\
\hline HYD5 & 9.07 & AC149033 & 3 & AC119747 & & & 1 & Sb01g048860 \\
\hline HYD6 & 1.01 & AC155364 & 10 & AE016959 & & & & \\
\hline CYP97C2 & 3.05 & BE552887 & 10 & AK065689 & $6 \mathrm{AL}, 6 \mathrm{BL}, 6 \mathrm{DL}$ & USDA & $1,146.3 \mathrm{cM}$ & Sb01g030050 \\
\hline CYP97A4 & 4 & AY112171 & 2 & AK068163 & & & 4 & Sb04g037300 \\
\hline ZEP1 & 2.04 & AC194845 & 4 & AL606448 & & & 6 & Sb06g018220 \\
\hline ZEP2 & 10.04 & AC206194 & NA & NA & & & & \\
\hline VDE & 2.05 & EU956472 & 1,4 & $\begin{array}{l}\text { Os01g0716400, } \\
\text { Os04g0379700 }\end{array}$ & & & 6 & Sb06g012950 \\
\hline NXS & & & 1 & Os01g03750 & & & 3 & Sb03g007320 \\
\hline CCD1 & 9.07 & AY106323 & 12 & Os12g44310 & & & $\begin{array}{l}1,48.5 \mathrm{cM} \text { qtl (color; } \\
p \leq 0.05)\end{array}$ & Sb01g047540 \\
\hline NCED1 & 1.08 & AC201886 & & & & & $1,210.3 \mathrm{cM}$ & \\
\hline NCED2 & 3.04 & AC199036 & 12 & Os12g42280 & & & 1 & Sbi_0.36552 \\
\hline NCED3a & & AC212820 & 7 & Os07g05940 & & & 2 & Sb02g003230 \\
\hline NCED3b & 2.09 & AC205109 & & & & & & \\
\hline
\end{tabular}


Table 2 | Continued

\begin{tabular}{|c|c|c|c|c|c|c|c|c|}
\hline \multirow[t]{2}{*}{ Enzyme } & \multicolumn{2}{|c|}{ Maize (Zea mays) } & \multicolumn{2}{|c|}{ Rice (Oryza sativa) } & \multicolumn{2}{|c|}{ Wheat (Triticum aestivum) } & \multicolumn{2}{|c|}{ Sorghum (Sorghum bicolor) } \\
\hline & Chr. bin & $\begin{array}{l}\text { Reference/ } \\
\text { GenBank }\end{array}$ & Chr. bin & $\begin{array}{l}\text { Reference/ } \\
\text { GenBank }\end{array}$ & Chr. bin & $\begin{array}{l}\text { Reference/ } \\
\text { GenBank }\end{array}$ & Chr. Bin and (qtl) & $\begin{array}{l}\text { Reference/ } \\
\text { phytozome }\end{array}$ \\
\hline CCD4a & 4.06 & AC190588 & 2 & Os02g47510 & & & 4 & Sb04g030640 \\
\hline $\operatorname{CCD} 4 \mathrm{~b}$ & 5.06 & AC194862 & 12 & Os12g24800 & & & & \\
\hline CCD7 & 2.03 & AC211432 & 4 & Os04g46470 & & & 6 & Sb06g024560 \\
\hline CCD8b & 7.02 & AC198395 & 9 & Os09g15240 & & & 2 & Sb02g021490; \\
\hline \multirow[t]{2}{*}{ CCD8c } & & & 1 & Os01g38580 & & & 10 & Sb10g004360 \\
\hline & & & & & & & 10 (8c-like) & Sb10g004370 \\
\hline \multirow[t]{2}{*}{ CCD8d } & & & 8 & Os08g28240 & & & 2 & Sb02g021490 \\
\hline & & & & & & & 7 (8d-like) & Sb07g024250 \\
\hline NCED9 & 5.03 & AC190614 & 3 & Os03g44380 & & & 1 & Sb01g013520 \\
\hline
\end{tabular}

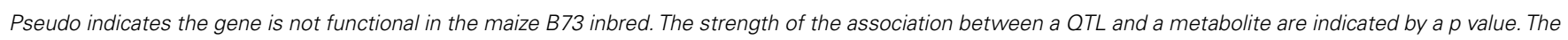

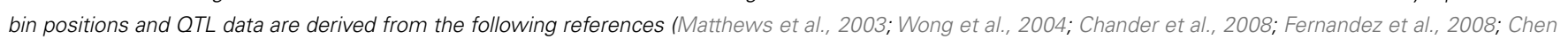
et al., 2010; Vallabhaneni et al., 2010; MaizeDatabase, www.maizegdb.org).

of genes controlling steps that supply isoprenoid precursors to the carotenoid pathway correlated with carotenoid content at 25 days after pollination, whereas the carotenoid pathway genes (PSY1, CrtISO, and ZEP) showed earlier correlation (20 days after pollination).

\section{QUANTITATIVE CONTROL OF CAROTENOID CONTENT THROUGH LIMITING DEGRADATION}

Carotenoid degradation is important in determining total carotenoid accumulation as well as composition. A family of carotenoid cleavage enzymes is required to process violaxanthin and neoxanthin into ABA and other pathway intermediates into an array of apocarotenoids. All of the maize cleavage genes have now been identified, mapped to chromosomes (Table 1), and compared with homologs from related species (Table 2; Vallabhaneni et al., 2010). Carotenoid cleavage can deplete the carotenoid pool, as was observed in Arabidopsis seeds and chrysanthemum flowers (Auldridge et al., 2006; Ohmiya et al., 2006). One maize catabolic gene, $Z m C C D 1$, was cloned and found to effectively cleave carotenoids at the 9, 10 position (Sun et al., 2008; Vogel et al., 2008). The position of $Z m C C D 1$, chromosome 9.07, is linked to the dominant white cap1 (wc1) locus (Vallabhaneni et al., 2010). Dominant $w c 1$ alleles exhibit reduced endosperm carotenoids, and there is evidence for a gene dosage effect as $Z m C C D 1$ transcript abundance negatively correlated with carotenoid content and an inbred line with high $Z m C C D 1$ copy number had concomitantly low endosperm carotenoid content (Vallabhaneni et al., 2010). Thus it is important to take advantage of the rich genetic resources to identify favorable CCD alleles that encourage retention of endosperm carotenoids.

\section{QUALITATIVE CONTROL OF CAROTENOID COMPOSITION}

Carotenoid composition is another important consideration as only carotenoids with unmodified $\beta$-ionone rings are converted to vitamin A. $\beta$-Carotene has the greatest provitamin A potential and it is therefore the preferred endosperm carotenoid for nutritional purposes. Optimizing $\beta$-carotene accumulation requires enhanced flux to the $\beta$-branch of the pathway in combination with limiting hydroxylation of $\beta$-carotene to downstream xanthophyll compounds that no longer have provitamin A activity.

The relative activities of the $\varepsilon$ - and $\beta$-cyclases alter flux to either branch of the pathway; LCYE activity leads to $\alpha$-carotene and lutein production at the expense of $\beta$-carotenoids. A diverse maize panel was subjected to association analysis, linkage mapping, and expression analyses showing that variation at the LCYE locus altered flux partitioning. Four polymorphisms were identified that controlled $58 \%$ of the variation between $\alpha$ - and $\beta$ branch accumulation, thus enabling selection of alleles that confer high provitamin A status for improved maize varieties (Harjes et al., 2008). This was a significant step in provitamin A enhancement, but still required the discovery of loci that were responsible for conversion of provitamin A $\beta$-carotene by hydroxylation to non-provitamin A products.

Breeding with select $L C Y E$ alleles to control increased flux through the $\beta$-branch of the pathway is only effective for biofortification if $\beta$-carotene remains unmodified by downstream hydroxylase enzymes. To identify target genes for blocking carotene hydroxylation, maize genes encoding carotene hydroxylases were investigated. Two structurally distinct classes of enzymes were found to be encoded by a total of eight genes in maize (Vallabhaneni et al., 2009). The gene families are similarly complex in other grasses. Using the maize diversity core collection produced by "metabolite sorting," it was possible to pinpoint the one carotene hydroxylase encoded by the Hydroxylase3 (HYD3) locus, whose transcript levels negatively correlated with high $\beta$ carotene levels and positively correlated with zeaxanthin levels. HYD3 was mapped close to a known QTL for $\beta$-carotene composition (see Table 1). PCR genotyping of 51 maize lines showed that the HYD3 locus could explain 36\% variation and fourfold difference in $\beta$-carotene levels (Vallabhaneni et al., 2009). Association and linkage population studies in maize confirmed that HYD3 was indeed responsible for this particular QTL associated 
with $\beta$-carotene accumulation (Yan et al., 2010). The most favorable alleles were found in temperate varieties and will be bred into tropical maize germplasm to help alleviate vitamin A deficiency in developing countries. Thus two significant gene targets to control $\beta$-carotene composition (LCYE and HYD3) have been identified, and the concomitant control of both of these steps promises to have a significant impact on provitamin A enhancement.

\section{OPEN QUESTIONS ABOUT PATHWAY REGULATION}

Carotenoid levels throughout the plant are regulated by developmental cues and various biotic and abiotic stresses (Cuttriss et al., 2011) In general, increases in carotenoid accumulation coincide with increased transcript abundance of some key (but not all) steps in the pathway; however no regulatory genes controlling carotenogenesis have been identified in maize or any other grass. As we tease apart the genetic diversity of maize carotenogenesis, it is hoped that we will identify transcription factors and feedback mechanisms that modulate carotenoid biosynthesis. Furthermore, the precise localization of carotenoid biosynthetic enzymes and their post-transcriptional interactions remain open questions along with unknown mechanisms of carotenoid sequestration in endosperm amyloplasts. Understanding protein import and interactions within the plastid will enable targeted manipulation of biosynthesis and more effective breeding strategies.

\section{LOOKING FORWARD TO OPTIMIZE $\beta$-CAROTENE LEVELS IN MAIZE}

Control points that determine carotenoid accumulation have produced promising molecular breeding tools, and while PSY has proven to be a major determinant, other biosynthetic and regulatory steps will have a significant impact on carotenogenesis. Understanding of allelic differences and new points of control can also be targeted in breeding projects. Certainly a molecular screen for a combination of preferable LCYE and HYD3 alleles will have a large impact on $\beta$-carotene synthesis and retention.

An additional promising approach that remains to be tested is the endosperm specific upregulation of isoprenoid precursor synthesis. Transcript abundance of several isoprenoid genes (DXS3, DXR, HDR, and GGPPS1) was found to positively correlate with endosperm carotenoid content (Vallabhaneni and Wurtzel, 2009) and thus those genes are potential biofortification targets.

\section{REFERENCES}

Aluru, M., Xu, Y., Guo, R., Wang, Z. G., Li, S. S., White, W., Wang, K., and Rodermel, S. (2008). Generation of transgenic maize with enhanced provitamin A content. J. Exp. Bot. 59, 3551-3562.

Auldridge, M. E., Mccarty, D. R., and Klee, H. J. (2006). Plant carotenoid cleavage oxygenases and their apocarotenoid products. Curr. Opin. Plant Biol. 9, 315-321.

Buckner, B., Kelson, T. L., and Robertson, D. S. (1990). Cloning of the y1 locus of maize, a gene involved in the biosynthesis of carotenoids. Plant Cell 2, 867-876.
Buckner, B., Miguel, P. S., Janickbuckner, D., and Bennetzen, J. L. (1996). The Y1 gene of maize codes for phytoene synthase. Genetics 143, 479-488.

Chander, S., Guo, Y. Q., Yang, X. H., Zhang, J., Lu, X. Q., Yan, J. B., Song, T. M., Rocheford, T. R., and Li, J. S. (2008). Using molecular markers to identify two major loci controlling carotenoid contents in maize grain. Theor. Appl. Genet. 116, 223-233.

Chaudhary, N., Nijhawan, A., Khurana, J. P., and Khurana, P. (2010). Carotenoid biosynthesis genes in rice: structural analysis, genome-wide expression

Manipulation of DXS3 is a particularly promising target, as DXS overexpression in Arabidopsis produced increased isoprenoids, including carotenoids (Estevez et al., 2001). Transgenic manipulations may offer the most expedient approach to control these additional targets given the absence of known regulatory factors to control multiple steps. Transgenic maize plants have been engineered to accumulate a wide variety of carotenoid intermediates and unusual keto-carotenoids and seeds ranged from white and yellow to dark-red, despite the white-endosperm genetic background (Aluru et al., 2008; Zhu et al., 2008). This achievement demonstrates remarkable plasticity in carotenoid accumulation and indicates that the targets identified by metabolite sorting and transcript profiling can be successfully manipulated.

Cereal breeding programs stand to be revolutionized with the increased availability of next generation and single-molecule sequencing technologies (Morozova and Marra, 2008; Edwards and Batley, 2010). The application of systems biology tools will enable us to expand analyses from candidate pathway genes to all genes in the genome, and to rapidly characterize germplasm diversity collections for crops that do not have the same wealth of genetic resources as maize. Proteomic and protein interaction analyses will precisely pinpoint where in the plastid carotenoid biosynthesis occurs. Combining these tools in an effective way will make for significant advances in metabolic engineering and biofortification of provitamin A carotenoids in cereal food crops. Improving the nutritional composition of such staples could have a positive impact on the health of millions of people worldwide.

\section{ACKNOWLEDGMENTS}

The authors acknowledge the support of current grants from the US National Institutes of Health (GM081160) to Eleanore T. Wurtzel, a FRST award from the New Zealand Foundation for Research Science and Technology (to Abby Cuttriss) and support from Professor David Christopher (to Abby Cuttriss). The carotenoid research on maize and other grasses that has been ongoing in the Wurtzel lab for over 25 years has been funded by the US National Institutes of Health, Rockefeller Foundation International Rice Biotechnology Program, McKnight Foundation, American Cancer Society, US National Science Foundation, United States Department of Agriculture, PSC-CUNY, and New York State.

profiling and phylogenetic analysis. MGG Mol. Genet. Genomics 283, 13-33.

Chen, Y., Li, F. Q., and Wurtzel, E. T. (2010). Isolation and characterization of the Z-ISO gene encoding a missing component of carotenoid biosynthesis in plants. Plant Physiol. 153, 66-79.

Cunningham, F. X., and Gantt, E. (2001). One ring or two? Determination of ring number in carotenoids by lycopene epsilon-cyclases. Proc. Natl. Acad. Sci. U.S.A. 98, 2905.

Cuttriss, A. J., Cazzonelli, C. I., Wurtzel, E. T., and Pogson, B. J. (2011) "Carotenoids," in Biosynthesis of
Vitamins in Plants Part A, Advances in Botanical Research, eds F. Rébeillé and R. Douce (Elsevier), 58, $1-36$.

Edwards, D., and Batley, J. (2010). Plant genome sequencing: applications for crop improvement. Plant Biotechnol. J. 8, 2-9.

Estevez, J. M., Cantero, A., Reindl, A., Reichler, S., and Leon, P. (2001). 1-Deoxy-D-xylulose-5-phosphate synthase, a limiting enzyme for plastidic isoprenoid biosynthesis in plants. J. Biol. Chem. 276, 22901-22909.

FAO. (2000). Food Insecurity: When People Live with Hunger and Fear Starvation. Rome: FAO. 
Fernandez, M. G. S., Hamblin, M. T., Li, L., Rooney, W. L., Tuinstra, M. R., and Kresovich, S. (2008). Quantitative trait loci analysis of endosperm color and carotenoid content in sorghum grain. Crop Sci. 48, 1732-1743.

Fernandez, M. G. S., Kapran, I., Souley, S., Abdou, M., Maiga, I. H., Acharya, C. B., Hamblin, M. T., and Kresovich, S. (2009). Collection and characterization of yellow endosperm sorghums from West Africa for biofortification. Genet. Resour. Crop Evol. 56, 991-1000.

Gallagher, C. E., Matthews, P. D., Li, F. Q., and Wurtzel, E. T. (2004). Gene duplication in the carotenoid biosynthetic pathway preceded evolution of the grasses. Plant Physiol. 135, 1776-1783.

Harjes, C. E., Rocheford, T. R., Bai, L., Brutnell, T. P., Kandianis, C. B., Sowinski, S. G., Stapleton, A. E., Vallabhaneni, R., Williams, M., Wurtzel, E. T., Yan, J. B., and Buckler, E. S. (2008). Natural genetic variation in lycopene epsilon cyclase tapped for maize biofortification. Science 319, 330-333.

Howitt, C. A., Cavanagh, C. R., Bowerman, A. F., Cazzonelli, C., Rampling, L., Mimica, J. L., and Pogson, B. J. (2009). Alternative splicing, activation of cryptic exons and amino acid substitutions in carotenoid biosynthetic genes are associated with lutein accumulation in wheat endosperm. Funct. Integr. Genomics 9, 363-376.

Joyard, J., Ferro, M., Masselon, C., Seigneurin-Berny, D., Salvi, D., Garin, J., and Rolland, N. (2009). Chloroplast proteomics and the compartmentation of plastidial isoprenoid biosynthetic pathways. Mol. Plant 2, 1154-1180.

Kirk, J. T. O., and Tiliney-Bassett, R. A. E. (1978). "Proplastids, etioplasts, amyloplasts, chromoplasts and other plastids," in The Plastids: Their Chemistry, Structure, Growth and Inheritance, eds J. T. O. Kirk and R. A. E. Tinley-Bassett (Amsterdam: Elsevier/North Holland Biomedical Press), 217-239.

Li, F., Vallabhaneni, R., and Wurtzel, E. T. (2008a). PSY3, a new member of the phytoene synthase gene family conserved in the Poaceae and regulator of abiotic-stress-induced root carotenogenesis. Plant Physiol. 146, 1333-1345.

Li, F. Q., Vallabhaneni, R., Yu, J., Rocheford, T., and Wurtzel, E. T. (2008b). The maize phytoene synthase gene family: Overlapping roles for carotenogenesis in endosperm, photomorphogenesis, and thermal stress tolerance. Plant Physiol. 147, 1334-1346.

Li, F. Q., Murillo, C., and Wurtzel, E. T. (2007). Maize Y9 encodes a product essential for 15-cis-zeta-carotene isomerization. Plant Physiol. 144, 1181-1189.

Li, F. Q., Tzfadia, O., and Wurtzel, E. T. (2009). The phytoene synthase gene family in the Grasses. Plant Signal. Behav. 4, 208-211.

Lichtenthaler, H. K. (1999). The 1-deoxy-d-xylulose-5-phosphate pathway of isoprenoid biosynthesis in plants. Annu. Rev. Plant Physiol. Plant Mol. Biol. 50, 47-65.

Liu, K., Goodman, M., Muse, S., Smith, J. S., Buckler, E., and Doebley, J. (2003). Genetic structure and diversity among maize inbred lines as inferred from DNA microsatellites. Genetics 165, 2117-2128.

Matthews, P. D., Luo, R., and Wurtzel, E. T. (2003). Maize phytoene desaturase and zetacarotene desaturase catalyze a poly- $Z$ desaturation pathway: implications for genetic engineering of carotenoid content among cereal crops. J. Exp. Bot. 54, 2215-2230.

Morozova, O., and Marra, M. A. (2008). Applications of next-generation sequencing technologies in functional genomics. Genomics 92, 255-264.

Neill, S. J., Horgan, R., and Parry, A. D. (1986). The carotenoid and abscisic acid content of viviparous kernels and seedlings of Zea mays L. Planta 169, 87-96.

North, H. M., De Almeida, A., Boutin, J. P., Frey, A., To, A., Botran, L., Sotta, B., and Marion-Poll, A. (2007). The Arabidopsis ABA-deficient mutant aba4 demonstrates that the major route for stress-induced ABA accumulation is via neoxanthin isomers. Plant J. 50, 810-824.

Ohmiya, A., Kishimoto, S., Aida, R., Yoshioka, S., and Sumitomo, K. (2006). Carotenoid cleavage dioxygenase $(\mathrm{CmCCD} 4 \mathrm{a})$ contributes to white color formation in Chrysanthemum petals. Plant Physiol. 142, 1193-1201.

Paine, J. A., Shipton, C. A., Chaggar, S., Howells, R. M., Kennedy, M. J., Vernon, G., Wright, S. Y., Hinchliffe, E., Adams, J. L., Silverstone, A. L., and Drake, R. (2005). Improving the nutritional value of golden rice through increased provitamin A content. Nat. Biotechnol. 23, 482-487.

Palaisa, K. A., Morgante, M., Williams, M., and Rafalski, A. (2003).
Contrasting effects of selection on sequence diversity and linkage disequilibrium at two phytoene synthase loci. Plant Cell 15, 1795-1806.

Randolph, L. F., and Hand, D. B. (1940). Relation between carotenoid content and number of genes per cell in diploid and tetraploid corn. J. Agric. Res. 60, 51-64.

Robertson, D., Bachmann, M., and Anderson, I. (1966). Role of carotenoids in protecting chlorophyll from photodestruction - II. Studies on the effect of four modifiers of the albino cll mutant of maize. Photochem. Photobiol. 5, 797-805.

Robertson, D. S. (1975). Survey of the albino and white-endosperm mutants of maize. J. Hered. 66 67-74.

Rodriguez-Concepcion, M. (2010). Supply of precursors for carotenoid biosynthesis in plants. Arch. Biochem. Biophys. 504, 118-122.

Singh, M., Lewis, P. E., Hardeman, K., Bai, L., Rose, J. K. C., Mazourek, M., Chomet, P., and Brutnell, T. P. (2003). Activator mutagenesis of the pink scutellum1/viviparous7 locus of maize. Plant Cell 15, 874-884.

Sommer, A., and Davidson, F. R. (2002). Assessment and control of vitamin A deficiency: the Annecy Accords. J. Nutr. 132, S2845-S2850.

Sun, Z., Hans, J., Walter, M. H. Matusova, R., Beekwilder, J., Verstappen, F. W. A., Ming, Z. Van Echtelt, E., Strack, D., Bisseling, T., and Bouwmeester, H. J. (2008). Cloning and characterisation of a maize carotenoid cleavage dioxygenase (ZmCCD1) and its involvement in the biosynthesis of apocarotenoids with various roles in mutualistic and parasitic interactions. Planta 228 789-801.

Treharne, K. J., Mercer, E. I., and Goodwin, T. W. (1966). Carotenoid biosynthesis in some maize mutants. Phytochemistry 5, 581-587.

Vallabhaneni, R., Bradbury, L. M. T., and Wurtzel,E. T. (2010). The carotenoid dioxygenase gene family in maize, sorghum, and rice. Arch. Biochem. Biophys. 504, 104-111.

Vallabhaneni, R., Gallagher, C. E., Licciardello, N., Cuttriss, A. J., Quinlan, R. F, and Wurtzel, E. T. (2009). Metabolite sorting of a germplasm collection reveals the Hydroxylase 3 locus as a new target for maize provitamin A biofortification. Plant Physiol. 151, 1635-1645.
Vallabhaneni, R., and Wurtzel, E. T. (2009). Timing and biosynthetic potential for carotenoid accumulation in genetically diverse germplasm of maize. Plant Physiol. 150, 562-572.

Vogel, J. T., Tan, B.-C., Mccarty, D. R., and Klee, H. J. (2008). The carotenoid cleavage dioxygenase 1 enzyme has broad substrate specificity, cleaving multiple carotenoids at two different bond positions. $J$. Biol. Chem. 283, 11364-11373.

Von Lintig, J. (2010). Colors with functions: elucidating the biochemical and molecular basis of carotenoid metabolism. Аnnu. Rev. Nutr. 30, 35-56.

Welsch, R., Wust, F., Bar, C., Al-Babili, S., and Beyer, P. (2008). A third phytoene synthase is devoted to abiotic stress-induced abscisic acid formation in rice and defines functional diversification of phytoene synthase genes. Plant Physiol. 147, $367-380$.

WHO. (2009). "Global prevalence of vitamin A deficiency in populations at risk 1995-2005," in WHO Global Database on Vitamin A Deficiency (Geneva: World Health Organization), 1-55.

Wong, J. C., Lambert, R. J., Wurtzel, E. T., and Rocheford, T. R. (2004). QTL and candidate genes phytoene synthase and zeta-carotene desaturase associated with the accumulation of carotenoids in maize. Theor. Appl. Genet. 108, 349-359.

Yan, J. B., Kandianis, C. B., Harjes, C. E., Bai, L., Kim, E. H., Yang, X. H., Skinner, D. J., Fu, Z. Y., Mitchell, S., Li, Q., Fernandez, M. G. S., Zaharieva, M., Babu, R., Fu, Y., Palacios, N., Li, J. S., Dellapenna, D., Brutnell, T., Buckler, E. S., Warburton, M. L., and Rocheford, T. (2010). Rare genetic variation at Zea mays crtRBl increases betacarotene in maize grain. Nat. Genet 42, 322-327.

Ye, X. D., Al-Babili, S., Kloti, A., Zhang, J., Lucca, P., Beyer, P., and Potrykus, I. (2000). Engineering the provitamin A (beta-carotene) biosynthetic pathway into (carotenoid-free) rice endosperm. Science 287, 303-305.

Ytterberg, A. J., Peltier, J. -B., and Van Wijk, K. J. (2006). Protein profiling of plastoglobules in chloroplasts and chromoplasts. A surprising site for differential accumulation of metabolic enzymes. Plant Physiol. 140, 984-997.

Zhu, C. F., Naqvi, S., Breitenbach, J., Sandmann, G., Christou, P., and Capell, T. (2008). Combinatorial 
genetic transformation generates a library of metabolic phenotypes for the carotenoid pathway in maize. Proc. Natl. Acad. Sci. U.S.A. 105, 18232-18237.

Conflict of Interest Statement: The authors declare that the research was conducted in the absence of any commercial or financial relationships that could be construed as a potential conflict of interest.

Received: 06 January 2012; paper pending published: 24 January 2012; accepted: 26 January 2012; published online: 20 February 2012.
Citation: Wurtzel ET, Cuttriss A and Vallabhaneni $R$ (2012) Maize provitamin A carotenoids, current resources, and future metabolic engineering challenges. Front. Plant Sci. 3:29. doi: 10.3389/fpls.2012.00029

This article was submitted to Frontiers in Plant Metabolism and Chemodiversity, a specialty of Frontiers in Plant Science.
Copyright (C) 2012 Wurtzel, Cuttriss and Vallabhaneni. This is an openaccess article distributed under the terms of the Creative Commons Attribution Non Commercial License, which permits non-commercial use, distribution, and reproduction in other forums, provided the original authors and source are credited. 